TRANSACTIONS OF THE

AMERICAN MATHEMATICAL SOCIETY

Volume 348, Number 8, August 1996

\title{
PACKING MEASURE OF THE SAMPLE PATHS OF FRACTIONAL BROWNIAN MOTION
}

\author{
YIMIN XIAO
}

\begin{abstract}
Let $X(t)(t \in \mathbf{R})$ be a fractional Brownian motion of index $\alpha$ in $\mathbf{R}^{d}$. If $1<\alpha d$, then there exists a positive finite constant $K$ such that with probability 1 ,$$
\phi-p(X([0, t]))=K t \quad \text { for any } t>0,
$$$$
\text { where } \phi(s)=s^{\frac{1}{\alpha}} /\left(\log \log \frac{1}{s}\right)^{\frac{1}{2 \alpha}} \text { and } \phi-p(X([0, t])) \text { is the } \phi \text {-packing measure of }
$$
$X([0, t])$.
\end{abstract}

\section{INTRODUCTION}

Packing measure was introduced by Taylor and Tricot ([TT]) as a dual concept to Hausdorff measure. Like Hausdorff measure and Hausdorff dimension (cf. [F]), it is a very useful tool in analyzing fractal sets and in studying sample path properties of stochastic processes. There has been a lot of work on the packing measure of the image and graph of processes with stationary independent increments (see [FT], $[\mathrm{LeT}],[\mathrm{RT}],[\mathrm{TT}]$, [Tay1], and references therein). The objective of this paper is to study the packing measure of the sample paths of fractional Brownian motion. For processes with stationary independent increments, the strong Markov property plays a crucial role. For this reason some of the techniques used previously are not available in the present situation.

Fix $\alpha \in(0,1)$ and consider the centered, real gaussian process $Y(t)(t \in \mathbf{R})$ with covariance

$$
E(Y(t) Y(s))=\frac{1}{2}\left(|t|^{2 \alpha}+|s|^{2 \alpha}-|t-s|^{2 \alpha}\right) .
$$

We will use the fact that $Y(t)(t \in \mathbf{R})$ can be represented as a stochastic integral

$$
Y(t)=\int_{\mathbf{R}} G(t, x) d W(x),
$$

where $d W(x)$ is a scattered gaussian random measure with Lebesgue measure as control measure and

$$
G(t, x)=c_{\alpha}\left(\max (t-x, 0)^{\alpha-\frac{1}{2}}-\max (-x, 0)^{\alpha-\frac{1}{2}}\right),
$$

Received by the editors August 2, 1995.

1991 Mathematics Subject Classification. Primary 60G15, 60G17.

Key words and phrases. Packing measure, fractional Brownian motion, image, sojourn time.

(c)1996 American Mathematical Society 
where $c_{\alpha}$ is a normalizing constant depending on $\alpha$ only. We associate with $Y$ a gaussian process in $\mathbf{R}^{d}$ by

$$
X(t)=\left(X_{1}(t), \cdots, X_{d}(t)\right),
$$

where $X_{1}, \cdots, X_{d}$ are independent copies of $Y$. Using the terminology of Kahane ([K], Chapter 18), we call $X$ the $(1, d, \alpha)$ process or the $d$-dimensional fractional Brownian motion of index $\alpha$. When $\alpha=\frac{1}{2}, X(t)$ is ordinary $d$-dimensional Brownian motion. It is easy to see that $X(t)(t \in \mathbf{R})$ is a self-similar process with exponent $\alpha$, i.e. for any $a>0$,

$$
X(a \cdot) \stackrel{\mathrm{d}}{=} a^{\alpha} X(\cdot),
$$

where $X \stackrel{\mathrm{d}}{=} Y$ means that the two processes $X$ and $Y$ have the same finite dimensional distributions.

It is well known that with probability 1 ,

$$
\operatorname{Dim} X([0,1])=\min \left\{d ; \frac{1}{\alpha}\right\},
$$

where $\operatorname{Dim} E$ is the packing dimension of $E$ (cf. [Tay2]). We shall prove that, in the transient case (that is, $1<\alpha d$ ), there exist positive constants $C_{1}$ and $C_{2}$, such that

$$
C_{1} \leq \phi-p(X([0,1])) \leq C_{2}, \quad \text { a.s. }
$$

where $\phi(s)=s^{\frac{1}{\alpha}} /\left(\log \log \frac{1}{s}\right)^{\frac{1}{2 \alpha}}$ and $\phi$ - $p$ is the $\phi$-packing measure. If $1>\alpha d$, then by a result of Pitt $[\mathrm{P}]$ (see also $[\mathrm{K}]$, Ch.18, Theorem 2 ), $X([0,1]$ ) a.s. contains interior points and hence $0<L_{d}(X([0,1]))<\infty$, a.s., where $L_{d}$ is the Lebesgue measure in $\mathbf{R}^{d}$. In the critical case of $1=\alpha d$, the problem of finding the packing measure of $X([0,1])$ is much deeper. Except in the special case of planar Brownian motion, for which LeGall and Taylor $([\mathrm{LeT}])$ proved that $\phi-p(X([0,1]))$ is either zero or infinite, this problem is still open.

The paper is organized as follows. In Section 2, we collect definitions and lemmas which are essential to our calculations. In Section 3, we obtain upper bounds for the probability that a fractional Brownian motion hits a ball, generalizing classical results for ordinary Brownian motion in $\mathbf{R}^{d}(d \geq 3)$. In Section 4 , we prove a lim inf theorem for the sojourn time of fractional Brownian motion. In Section 5, we prove (1.4). We have to use methods quite different from those for Brownian motion to prove our results. The reason for this is not only that for $\alpha \neq \frac{1}{2}$ fractional Brownian motion of index $\alpha$ does not have independent increments; there are also other technical difficulties. For example, the distribution of the sojourn time of $d$ dimensional Brownian motion in the unit ball $\mathbf{R}^{d}$ is explicitly known ([CT], [TT]) but for fractional Brownian motion of index $\alpha \neq \frac{1}{2}$, we can not even prove that the sojourn time has a bounded density.

We will use $K, K_{1}, K_{2}, \cdots, c_{1}, c_{2}, \cdots$ to denote unspecified positive finite constants which may take different values from line to line.

\section{Preliminaries}

First we recall briefly the definitions of packing measure and packing dimension. Let $\Phi$ be the class of functions $\phi:(0, \delta) \rightarrow(0,1)$ which are right continuous, 
monotone increasing with $\phi(0+)=0$, and such that there exists a finite constant $K_{1}>0$ for which

$$
\frac{\phi(2 s)}{\phi(s)} \leq K_{1} \quad \text { for } 0<s<\frac{1}{2} \delta .
$$

For $\phi \in \Phi$, Taylor and Tricot ([TT]) defined the set function $\phi-P(E)$ on $\mathbf{R}^{N}$ by

$$
\phi-P(E)=\lim _{\epsilon \rightarrow 0} \sup \left\{\sum_{i} \phi\left(2 r_{i}\right): \bar{B}\left(x_{i}, r_{i}\right) \text { are disjoint, } x_{i} \in E, r_{i}<\epsilon\right\},
$$

where $B(x, r)$ denotes the open ball of radius $r$ centered at $x$. A sequence of closed balls satisfying the conditions in the right hand side of (2.2) is called an $\epsilon$-packing of $E$. Observe that $\phi-P$ is not an outer measure because it fails to be countably subadditive. However, $\phi-P$ is a premeasure, so one can obtain a metric outer measure $\phi-p$ on $\mathbf{R}^{N}$ by defining

$$
\phi-p(E)=\inf \left\{\sum_{n} \phi-P\left(E_{n}\right): E \subseteq \bigcup_{n} E_{n}\right\} .
$$

$\phi-p(E)$ is called the $\phi$-packing measure of $E$. Every Borel set in $\mathbf{R}^{N}$ is $\phi-p$ measurable. If $\phi(s)=s^{\alpha}, s^{\alpha}-p(E)$ is called the $\alpha$-dimensional packing measure of $E$. The packing dimension of $E$ is defined by

$$
\begin{aligned}
\operatorname{Dim} E & =\inf \left\{\alpha>0: \quad s^{\alpha}-p(E)=0\right\} \\
& =\sup \left\{\alpha>0: \quad s^{\alpha}-p(E)=+\infty\right\} .
\end{aligned}
$$

By (2.3), we see that, for any $E \subset \mathbf{R}^{N}$,

$$
\phi-p(E) \leq \phi-P(E) .
$$

The upper bound for $\phi-P(E)$ is usually not easy to determine, because we need to consider all the possible packings in (2.2). A lower bound for $\phi-p(E)$ can be obtained by using the following density theorem for packing measures (see [TT] and $[\mathrm{RT}]$ for a proof).

Lemma 2.1. Let $\mu$ be a Borel measure on $\mathbf{R}^{N}$ and $\phi \in \Phi$. Then for any Borel set $E \subseteq \mathbf{R}^{N}$,

$$
\phi-p(E) \geq K_{1}^{-3} \mu(E) \inf _{x \in E}\left\{\underline{D}_{\mu}^{\phi}(x)\right\}^{-1},
$$

where $K_{1}$ is the constant in (2.1) and

$$
\underline{D}_{\mu}^{\phi}(x)=\liminf _{r \rightarrow 0} \frac{\mu(B(x, r))}{\phi(2 r)}
$$

is the lower $\phi$-density of $\mu$ at $x$.

Now we recall some facts about gaussian processes. Let $Y(t)(t \in S)$ be a gaussian process. We define a metric $d$ on $S$ by

$$
d(s, t)=\left(E(Y(s)-Y(t))^{2}\right)^{\frac{1}{2}} .
$$

Denote by $N_{d}(S, \epsilon)$ the smallest number of open $d$-balls of radius $\epsilon$ needed to cover $S$, and write $D=\sup \{d(s, t): s, t \in S\}$.

The following lemma is well known. It is a consequence of the gaussian isoperimetric inequality and Dudley's entropy bound ([LT], see also [Ta1]). We will use it when $S$ is an interval. 
Lemma 2.2. There exists an absolute constant $K>0$ such that for any $u>0$, we have

$$
P\left\{\sup _{s, t \in S}|Y(s)-Y(t)| \geq K\left(u+\int_{0}^{D} \sqrt{\log N_{d}(S, \epsilon)} d \epsilon\right)\right\} \leq \exp \left(-\frac{u^{2}}{D^{2}}\right) .
$$

The following lemma is a corollary of Lemma 2.2, and (ii) is proved in $[\mathrm{M}]$.

Lemma 2.3. Let $Y(t)(t \in \mathbf{R})$ be a centered gaussian process with values in $\mathbf{R}$ such that $Y(0)=0$,

$$
E(Y(t)-Y(s))^{2} \leq \theta^{2}|t-s|^{2 \alpha} \quad(0<\alpha<1) .
$$

Then:

(i) For any $r>0, u \geq K r^{\alpha}$, we have

$$
P\left\{\sup _{|t| \leq r}|Y(t)| \geq \theta u\right\} \leq \exp \left(-\frac{u^{2}}{K r^{2 \alpha}}\right) .
$$

(ii) Let

$$
\omega_{Y}(h)=\sup _{t, t+s \in[0,1],|s| \leq h}|Y(t+s)-Y(t)|
$$

be the uniform modulus of continuity of $Y(t)$ on $[0,1]$. Then

$$
\limsup _{h \rightarrow 0} \frac{\omega_{Y}(h)}{\theta h^{\alpha}\left(\log \frac{1}{h}\right)^{\frac{1}{2}}} \leq K_{2}, \quad \text { a.s. }
$$

Let $\left\{A_{k}\right\}$ be a sequence of events in a probability space. In the following lemma, (i) is well known and (ii) is proved implicitly in [M] (see also [Ta2]).

Lemma 2.4. (i) If $\sum_{k=1}^{\infty} P\left(A_{k}\right)<\infty$, then $P\left(\lim \sup _{k \rightarrow \infty} A_{k}\right)=0$.

(ii) If there exist positive constants $K, \epsilon$ and positive integers $k_{0}, J$ such that for $k_{0} \leq k<J$,

$$
\sum_{j=k+1}^{J} P\left(A_{k} \cap A_{j}\right) \leq P\left(A_{k}\right)\left(K+(1+\epsilon) \sum_{j=k+1}^{J} P\left(A_{j}\right)\right)
$$

and

$$
\sum_{k=k_{0}}^{J} P\left(A_{k}\right) \geq \frac{1+2 K}{\epsilon}
$$

then

$$
P\left(\bigcup_{k=k_{0}}^{J} A_{k}\right) \geq \frac{1}{1+2 \epsilon}
$$

Remark. If $\sum_{k} P\left(A_{k}\right)=\infty$, then for each fixed $k_{0}$, we can take $J$ large enough so that (2.6) holds. 


\section{Hitting Probabilities}

In this section, we consider the probability that a $d$-dimensional fractional Brownian motion of index $\alpha$ hits a ball $B(y, r)$ in $\mathbf{R}^{d}$. The main results are Theorems 3.1 and 3.2, which generalize the classical results about the hitting probability and delayed hitting probability of $d$-dimensional Brownian motion $(d \geq 3)$.

Let $Z(t)(t \in \mathbf{R})$ be a centered, real valued gaussian process. We write

$$
\sigma^{2}(t, s)=E(Z(t)-Z(s))^{2}, \quad \sigma^{2}(t)=E(Z(t))^{2} .
$$

Suppose that

$$
\sigma^{2}(t, s) \leq \theta^{2}|t-s|^{2 \alpha} \quad(0<\alpha<1)
$$

and there exist positive constants $c_{1}, c_{2}$ such that

$$
c_{1} \theta^{2} t^{2 \alpha} \leq \sigma^{2}(t) \leq c_{2} \theta^{2} t^{2 \alpha} .
$$

Let $Y_{1}, \cdots, Y_{d}$ be independent copies of $Z$ and let

$$
Y(t)=\left(Y_{1}(t), \cdots, Y_{d}(t)\right) .
$$

Lemma 3.1. For any $a>0$, let $S=[a, 2 a]$. Consider a centered real gaussian process $Z(t)(t \in \mathbf{R})$ that satisfies (3.1) and (3.2) on $S$, where $\theta$ may depend on a. Let $Y(t)(t \in \mathbf{R})$ be the associated gaussian process in $\mathbf{R}^{d}$. Then there exist positive constants $K_{3}$ and $K_{4}$, depending only on $\alpha$ and $d$, such that for any $r>0$ and any $y \in \mathbf{R}^{d}$ with $|y|>K_{3} r$, we have

$$
P\left\{\inf _{t \in S}|Y(t)-y|<r\right\} \leq K_{4} \exp \left(-\frac{|y|^{2}}{K_{4} \theta^{2} a^{2 \alpha}}\right) \cdot a^{1-\alpha d} \cdot\left(\frac{r}{\theta}\right)^{d-\frac{1}{\alpha}} .
$$

Proof. Denote by $N\left(S,\left(\frac{r}{\theta}\right)^{1 / \alpha}\right)$ the smallest number of open balls of radius $\left(\frac{r}{\theta}\right)^{1 / \alpha}$ that are needed to cover $S$. Then

$$
N\left(S,\left(\frac{r}{\theta}\right)^{1 / \alpha}\right) \leq a \cdot\left(\frac{r}{\theta}\right)^{-\frac{1}{\alpha}},
$$

Let $\left\{S_{p}\right\}\left(1 \leq p \leq N\left(S,\left(\frac{r}{\theta}\right)^{1 / \alpha}\right)\right)$ be a family of balls of radius $\left(\frac{r}{\theta}\right)^{1 / \alpha}$ that cover $S$ and let

$$
\begin{aligned}
A & =\left\{\inf _{t \in S}|Y(t)-y|<r\right\}, \\
A_{p} & =\left\{\inf _{t \in S_{p}}|Y(t)-y|<r\right\} .
\end{aligned}
$$

Then

$$
A \subseteq \bigcup_{p=1}^{N\left(S,\left(\frac{r}{\theta}\right)^{1 / \alpha}\right)} A_{p} .
$$

Now fix a $1 \leq p \leq N\left(S,\left(\frac{r}{\theta}\right)^{1 / \alpha}\right)$. Let

$$
\epsilon_{n}=\left(\frac{r}{\theta}\right)^{\frac{1}{\alpha}} \exp \left(-2^{n+1}\right)
$$

and let $\left\{t_{i}^{(n)}, 1 \leq i \leq N\left(S_{p}, \epsilon_{n}\right)\right\}$ be a set of the centers of open balls with radius $\epsilon_{n}$ that cover $S_{p}$. Denote

$$
r_{n}=\beta \theta d \epsilon_{n}^{\alpha} 2^{\frac{n+1}{2}}
$$


where $\beta \geq K_{2}$ is a constant to be determined later, and

$$
\begin{aligned}
A_{i}^{(j)} & =\left\{\left|Y\left(t_{i}^{(j)}\right)-y\right| \leq r+\sum_{k=j}^{\infty} r_{k}\right\}, \\
A^{(n)} & =\bigcup_{j=1}^{n} \bigcup_{i=1}^{N\left(S_{p}, \epsilon_{j}\right)} A_{i}^{(j)} \\
& =A^{(n-1)} \cup \bigcup_{i=1}^{N\left(S_{p}, \epsilon_{n}\right)} A_{i}^{(n)} .
\end{aligned}
$$

Then by Lemma 2.3 (ii), we have

$$
P\left(A_{p}\right) \leq \lim _{n \rightarrow \infty} P\left(A^{(n)}\right) .
$$

By (3.6), we have

$$
P\left(A^{(n)}\right) \leq P\left(A^{(n-1)}\right)+P\left(A^{(n)} \backslash A^{(n-1)}\right)
$$

and

$$
P\left(A^{(n)} \backslash A^{(n-1)}\right) \leq \sum_{i=1}^{N\left(S_{p}, \epsilon_{n}\right)} P\left(A_{i}^{(n)} \backslash A_{i^{\prime}}^{(n-1)}\right),
$$

where $i^{\prime}$ is chosen so that $\left|t_{i}^{(n)}-t_{i^{\prime}}^{(n-1)}\right|<\epsilon_{n-1}$. Consider

$$
\begin{aligned}
& P\left(A_{i}^{(n)} \backslash A_{i^{\prime}}^{(n-1)}\right) \\
& =P\left\{\left|Y\left(t_{i}^{(n)}\right)-y\right|<r+\sum_{k=n}^{\infty} r_{k},\left|Y\left(t_{i^{\prime}}^{(n-1)}\right)-y\right|>r+\sum_{k=n-1}^{\infty} r_{k}\right\} \\
& \leq P\left\{\left|Y\left(t_{i}^{(n)}\right)-y\right|<r+\sum_{k=n}^{\infty} r_{k},\left|Y\left(t_{i}^{(n)}\right)-Y\left(t_{i^{\prime}}^{(n-1)}\right)\right| \geq r_{n-1}\right\} .
\end{aligned}
$$

By elementary properties of gaussian variables, we can write

$$
\frac{Z\left(t_{i}^{(n)}\right)-Z\left(t_{i^{\prime}}^{(n-1)}\right)}{\sigma\left(t_{i}^{(n)}, t_{i^{\prime}}^{(n-1)}\right)}=\rho \frac{Z\left(t_{i}^{(n)}\right)}{\sigma\left(t_{i}^{(n)}\right)}+\sqrt{1-\rho^{2}} \xi,
$$

where

$$
\rho=\frac{E\left[\left(Z\left(t_{i}^{(n)}\right)-Z\left(t_{i^{\prime}}^{(n-1)}\right)\right) Z\left(t_{i}^{(n)}\right)\right]}{\sigma\left(t_{i}^{(n)}, t_{i^{\prime}}^{(n-1)}\right) \sigma\left(t_{i}^{(n)}\right)}
$$

and where $\xi$ is a centered gaussian variable with variance 1 and is independent of $Z\left(t_{i}^{(n)}\right)$. Hence there exists a centered gaussian vector $\Xi$ with the identity matrix as its covariance matrix such that $\Xi$ is independent of $Y\left(t_{i}^{(n)}\right)$ and

$$
\frac{Y\left(t_{i}^{(n)}\right)-Y\left(t_{i^{\prime}}^{(n-1)}\right)}{\sigma\left(t_{i}^{(n)}, t_{i^{\prime}}^{(n-1)}\right)}=\rho \frac{Y\left(t_{i}^{(n)}\right)}{\sigma\left(t_{i}^{(n)}\right)}+\sqrt{1-\rho^{2}} \Xi .
$$


We observe that

$$
\begin{aligned}
r+\sum_{k=n}^{\infty} r_{k} & \leq r+\sum_{k=0}^{\infty} r_{k} \\
& \leq\left(1+c_{3} \int_{0}^{\infty} \exp \left(-\alpha x^{2}\right) d x\right) r \\
& =c_{4} r .
\end{aligned}
$$

and by (3.1),

$$
\sigma\left(t_{i}^{(n)}, t_{i^{\prime}}^{(n-1)}\right) \leq \theta\left|t_{i}^{(n)}-t_{i^{\prime}}^{(n-1)}\right|^{\alpha} .
$$

It follows that (3.10) is less than

$$
\begin{aligned}
& P\left\{\left|Y\left(t_{i}^{(n)}\right)-y\right| \leq c_{4} r,|\Xi| \geq \frac{1}{\sqrt{1-\rho^{2}}}\left[\frac{r_{n-1}}{\theta\left|t_{i}^{(n)}-t_{i^{\prime}}^{(n-1)}\right|^{\alpha}}-\rho \frac{Y\left(t_{i}^{(n)}\right)}{\sigma\left(t_{i}^{(n)}\right)}\right]\right\} \\
& \leq P\left\{\left|Y\left(t_{i}^{(n)}\right)-y\right| \leq c_{4} r,|\Xi| \geq \frac{\beta d}{2} 2^{\frac{n}{2}}\right\} \\
& \quad+P\left\{\left|Y\left(t_{i}^{(n)}\right)-y\right| \leq c_{4} r, \rho \frac{\left|Y\left(t_{i}^{(n)}\right)\right|}{\sigma\left(t_{i}^{(n)}\right)} \geq \frac{\beta d}{2} 2^{\frac{n}{2}}\right\} \\
& \hat{=} I_{1}+I_{2} .
\end{aligned}
$$

By the independence of $\Xi$ and $Y\left(t_{i}^{(n)}\right)$, we have

$$
\begin{aligned}
I_{1} & =P\left\{\left|Y\left(t_{i}^{(n)}\right)-y\right| \leq c_{4} r\right\} \cdot P\left\{|\Xi| \geq \frac{\beta d}{2} 2^{\frac{n}{2}}\right\} \\
& =\int_{\left\{|u-y| \leq c_{4} r\right\}} \frac{1}{(2 \pi)^{d / 2} \sigma^{d}\left(t_{i}^{(n)}\right)} \exp \left(-\frac{|u|^{2}}{2 \sigma^{2}\left(t_{i}^{(n)}\right)}\right) d u \cdot P\left\{|\Xi| \geq \frac{\beta d}{2} 2^{\frac{n}{2}}\right\} \\
& \leq c_{5} \exp \left(-\frac{|y|^{2}}{4 \sigma^{2}\left(t_{i}^{(n)}\right)}\right)\left(\frac{r}{\sigma\left(t_{i}^{(n)}\right)}\right)^{d} \cdot P\left\{|\Xi| \geq \frac{\beta d}{2} 2^{\frac{n}{2}}\right\} \quad\left(\text { if }|y| \geq K_{3} r\right) \\
& \leq c_{6} \exp \left(-\frac{|y|^{2}}{4 c_{2} \theta^{2}(2 a)^{2 \alpha}}\right)\left(\frac{r}{c_{1} \theta a^{\alpha}}\right)^{d} \cdot \exp \left(-\frac{(\beta d)^{2}}{16} 2^{n}\right),
\end{aligned}
$$

where the last inequality follows from (3.2) and the tail probability of the gaussian vector. On the other hand,

$$
\begin{aligned}
I_{2} & \leq \int_{\left\{|u-y| \leq c_{4} r,|u| \geq \frac{\beta d}{2} 2^{n / 2} \sigma\left(t_{i}^{(n)}\right)\right\}}\left(\frac{1}{2 \pi}\right)^{\frac{d}{2}} \frac{1}{\sigma^{d}\left(t_{i}^{(n)}\right)} \exp \left(-\frac{|u|^{2}}{2 \sigma^{2}\left(t_{i}^{(n)}\right)}\right) d u \\
& \leq c_{7} \int_{\left\{|u-y| \leq c_{4} r\right\}} \frac{1}{\sigma^{d}\left(t_{i}^{(n)}\right)} \exp \left(-\frac{|u|^{2}}{4 \sigma^{2}\left(t_{i}^{(n)}\right)}\right) d u \cdot \exp \left(-\frac{(\beta d)^{2}}{16} 2^{n}\right) \\
& \leq c_{8} \exp \left(-\frac{|y|^{2}}{8 \sigma^{2}\left(t_{i}^{(n)}\right)}\right)\left(\frac{r}{\sigma\left(t_{i}^{(n)}\right)}\right)^{d} \cdot \exp \left(-\frac{(\beta d)^{2}}{16} 2^{n}\right) \quad\left(\text { if }|y| \geq K_{3} r\right) \\
& \leq c_{9} \exp \left(-\frac{|y|^{2}}{8 c_{2} \theta^{2}(2 a)^{2 \alpha}}\right)\left(\frac{r}{c_{1} \theta a^{\alpha}}\right)^{d} \cdot \exp \left(-\frac{(\beta d)^{2}}{16} 2^{n}\right) .
\end{aligned}
$$

Combining (3.8) through (3.13) and choosing $\beta \geq K_{2}$ satisfying

$$
\frac{(\beta d)^{2}}{16}>2
$$


we obtain

$$
\begin{aligned}
P\left(A^{(n)}\right) \leq & P\left(A^{(n-1)}\right)+c_{10} N\left(S_{p}, \epsilon_{n}\right) \exp \left(-\frac{|y|^{2}}{8 c_{2} \theta^{2}(2 a)^{2 \alpha}}\right)\left(\frac{r}{\theta a^{\alpha}}\right)^{d} \\
& \cdot \exp \left(-\frac{(\beta d)^{2}}{16} 2^{n}\right) \\
\leq & c_{11}\left[N\left(S_{p}, \epsilon_{0}\right)+\sum_{k=1}^{\infty} N\left(S_{p}, \epsilon_{k}\right) \exp \left(-\frac{(\beta d)^{2}}{16} 2^{k}\right)\right] \\
& \cdot \exp \left(-\frac{|y|^{2}}{8 c_{2} \theta^{2}(2 a)^{2 \alpha}}\right)\left(\frac{r}{\theta a^{\alpha}}\right)^{d} \\
\leq & c_{12} \exp \left(-\frac{|y|^{2}}{8 c_{2} \theta^{2}(2 a)^{2 \alpha}}\right)\left(\frac{r}{\theta a^{\alpha}}\right)^{d} .
\end{aligned}
$$

Therefore, by (3.4), (3.5) and (3.7), we have

$$
P\left\{\inf _{t \in S}|Y(t)-y| \leq r\right\} \leq c_{13} \exp \left(-\frac{|y|^{2}}{8 c_{2} \theta^{2}(2 a)^{2 \alpha}}\right) \cdot \frac{1}{a^{\alpha d-1}} \cdot\left(\frac{r}{\theta}\right)^{d-\frac{1}{\alpha}} .
$$

This proves (3.3) with $K_{4}=\max \left(8 c_{2} 2^{2 \alpha} ; c_{13}\right)$.

Theorem 3.1. Let $X(t)(t \in \mathbf{R})$ be a d-dimensional fractional Brownian motion of index $\alpha$. If $1<\alpha d$, then there exists a positive constant $K$, depending only on $\alpha$ and $d$, such that for any $r>0$ and any $y \in \mathbf{R}^{d}$ with $|y| \geq K_{3} r$, we have

$$
P\{\exists t>0 \text { such that }|X(t)-y|<r\} \leq K\left(\frac{r}{|y|}\right)^{d-\frac{1}{\alpha}} .
$$

Proof. Clearly, $X(t)(t \in \mathbf{R})$ satisfies (3.1) and (3.2) with $\theta=1$. For any $n \in \mathbf{Z}$, let $S_{n}=\left[2^{n}, 2^{n+1}\right]$. Then

$$
(0, \infty)=\bigcup_{n \in \mathbf{Z}} S_{n}
$$

By Lemma 3.1, we have

$$
\begin{aligned}
& P\{\exists t>0 \text { such that }|X(t)-y|<r\} \\
& \quad \leq \sum_{n \in \mathbf{Z}} P\left\{\inf _{t \in S_{n}}|X(t)-y|<r\right\} \\
& \quad \leq K_{4} \sum_{n \in \mathbf{Z}} \exp \left(-\frac{|y|^{2}}{K_{4} 2^{2 \alpha n}}\right) \cdot 2^{n(1-\alpha d)} \cdot r^{d-\frac{1}{\alpha}} \\
& \quad \leq c_{14} \int_{0}^{\infty} \frac{1}{x^{\alpha d}} \exp \left(-\frac{|y|^{2}}{K_{4} x^{2 \alpha}}\right) d x \cdot r^{d-\frac{1}{\alpha}} \\
& \quad \leq K\left(\frac{r}{|y|}\right)^{d-\frac{1}{\alpha}} .
\end{aligned}
$$

This proves (3.14).

Remark. If $X(t)\left(t \in \mathbf{R}_{+}\right)$is a Brownian motion in $\mathbf{R}^{d}$ with $d \geq 3$, then it is well known that (3.14) holds with equality and $K=1$. This result was stated by Kakutani in 1944 ([Ka]) for $d=3$. For a proof of the general result, see [PS]. 
The following theorem is a generalization of the delayed hitting probability of Brownian motion in $\mathbf{R}^{d}(d \geq 3)$ in $[\mathrm{DE}]$.

Theorem 3.2. Let $X(t)(t \in \mathbf{R})$ be a d-dimensional fractional Brownian motion of index $\alpha(0<\alpha<1)$ with $1<\alpha d$. Then for any $T>0$ and any $0<r<T^{\alpha}$, we have

$$
P\{\exists t \in \mathbf{R} \text { such that }|t|>T \text { and }|X(t)|<r\} \leq K\left(\frac{r}{T^{\alpha}}\right)^{d-\frac{1}{\alpha}},
$$

where $K>0$ is a constant depending only on $\alpha$ and $d$.

Proof. Observing that the distribution of $X(T)$ and $X(-T)$ has the density

$$
p(x, T)=\left(\frac{1}{2 \pi}\right)^{\frac{d}{2}} \frac{1}{T^{\alpha d}} \cdot \exp \left(-\frac{|x|^{2}}{2 T^{2 \alpha}}\right),
$$

we have

$$
\begin{aligned}
& P\{\exists t \in \mathbf{R} \text { such that }|t|>T \text { and }|X(t)|<r\} \\
& \leq 2 P\{\exists t>T \text { such that }|X(t)|<r\} \\
& =2 \int_{\mathbf{R}^{d}} P\{\exists t>T \text { such that }|X(t)|<r \mid X(T)=y\} p(y, T) d y
\end{aligned}
$$

Using conditional expectation, for any $t>0$, we can write

$$
X(t T+T)=X_{1}(t)+c(t) X(T),
$$

where $X_{1}$ is independent of $X(T)$ and

$$
c(t)=\frac{(1+t)^{2 \alpha}+1-t^{2 \alpha}}{2} .
$$

It is clear that $c(t) \geq \frac{1}{2}$ and $c(t) \equiv 1$ if $\alpha=\frac{1}{2} ; c(t)$ is decreasing if $\alpha<\frac{1}{2}$ and $c(t)$ is increasing if $\alpha>\frac{1}{2}$. Moreover, there exists a constant $c_{15}>0$ such that

$$
|c(t)-c(s)| \leq c_{15}|t-s|^{\gamma},
$$

where $\gamma=2 \alpha$ if $\alpha \leq \frac{1}{2} ; \gamma=1$ if $\alpha>\frac{1}{2}$. In order to estimate the conditional probability in (3.16), let

$$
Y(t)=\frac{X_{1}(t)}{c(t)} .
$$

Now we verify that $Y(t)$ satisfies the conditions in Lemma 3.1. By (3.17), (3.18) and elementary calculations, we have

$$
\begin{aligned}
\sigma^{2}(t, s) & =T^{2 \alpha} \frac{|t-s|^{2 \alpha}}{c(t) c(s)}+T^{2 \alpha} \frac{c(t)-c(s)}{c(t) c(s)}\left(\frac{(1+s)^{2 \alpha}}{c(s)}-\frac{(1+t)^{2 \alpha}}{c(t)}\right) \\
& \leq \frac{c_{16} T^{2 \alpha}}{c(t) c(s)}|t-s|^{2 \alpha}, \\
\sigma^{2}(t) & =\frac{T^{2 \alpha}}{c^{2}(t)}\left[(1+t)^{2 \alpha}-\frac{\left((1+t)^{2 \alpha}+1-t^{2 \alpha}\right)^{2}}{4}\right],
\end{aligned}
$$


and (3.2) holds on any $S=[a, 2 a]$ with

$$
\theta=\frac{T^{\alpha}}{m}, \quad \text { where } m=\min \{c(a), c(2 a)\} .
$$

Hence by Lemma 3.1, for any $r>0$ and for any $y \in \mathbf{R}^{d}$ with $|y| \geq K_{3} r$, we have

$$
\begin{aligned}
& P\left\{\inf _{t \in S}\left|X_{1}(t)-c(t) y\right|<r\right\} \\
& \leq P\left\{\inf _{t \in S}|Y(t)-y|<\frac{r}{m}\right\} \\
& \leq K_{4} \exp \left(-\frac{|y|^{2} m^{2}}{K_{4} T^{2 \alpha} a^{2 \alpha}}\right) a^{1-\alpha d} \cdot\left(\frac{r}{T^{\alpha}}\right)^{d-\frac{1}{\alpha}} .
\end{aligned}
$$

By using the same argument as in the proof of Theorem 3.1, we obtain that for $|y| \geq K_{3} r$

$$
P\left\{\exists t>0 \text { such that }\left|X_{1}(t)+c(t) y\right|<r\right\} \leq K\left(\frac{r}{|y|}\right)^{d-\frac{1}{\alpha}} .
$$

Putting (3.20) into (3.16), we have

$$
\begin{aligned}
(3.16) & \leq 2 \int_{\mathbf{R}^{d}} \min \left\{1, K\left(\frac{r}{|y|}\right)^{d-\frac{1}{\alpha}}\right\} \cdot p(y, T) d y \\
& \leq 2 \int_{\left\{|y| \leq c_{17} r\right\}} p(y, T) d y+2 \int_{\left\{|y| \geq c_{17} r\right\}} K\left(\frac{r}{|y|}\right)^{d-\frac{1}{\alpha}} p(y, T) d y \\
& \leq c_{18}\left(\frac{r}{T^{\alpha}}\right)^{d}+c_{19}\left(\frac{r}{T^{\alpha}}\right)^{d-\frac{1}{\alpha}} \\
& \leq K\left(\frac{r}{T^{\alpha}}\right)^{d-\frac{1}{\alpha}}
\end{aligned}
$$

This completes the proof of (3.15).

Dvoretzky and Erdös ([DE] $)$ proved that if $X(t)$ is $d$-dimensional Brownian motion with $d \geq 3$, then the upper bound in (3.15) also serves as a lower bound (with a different constant). We have not been able to prove an analogous result for fractional Brownian motion, so we pose the following

Question. Are the upper bounds in Theorems 3.1 and 3.2 the best possible?

\section{A Limit Theorem For the Sojourn Time}

In this section, we prove a liminf theorem for the sojourn time of fractional Brownian motion in the ball $B(0, r)$. In next section, we will use the result to calculate the packing measure of the sample paths of a transient fractional Brownian motion.

For any $r>0$ and any $y \in \mathbf{R}^{d}$, let

$$
T_{y}(r)=\int_{\mathbf{R}} 1_{B(y, r)}(X(t)) d t
$$


be the sojourn time of $X(t)(t \in \mathbf{R})$ in $B(y, r)$. If $y=0$, we denote $T_{y}(r)$ by $T(r)$. By the self-similarity of $X(t)$, we have for any $a>0$,

$$
T(a \cdot) \stackrel{\mathrm{d}}{=} a^{\frac{1}{\alpha}} T(\cdot) .
$$

Lemma 4.1. For any $0<u<1$,

$$
\exp \left(-\frac{K}{u^{2 \alpha}}\right) \leq P\{T(1) \leq u\} \leq \exp \left(-\frac{1}{K u^{2 \alpha}}\right),
$$

where $K>0$ is a constant depending only on $\alpha$ and $d$.

Proof. The right inequality in (4.2) is easy:

$$
\begin{aligned}
P\{T(1) \leq u\} & \leq P\left\{\max _{t \in[0, u]}|X(t)| \geq 1\right\} \\
& =P\left\{\max _{t \in[0,1]}|X(t)| \geq u^{-\alpha}\right\} \\
& \leq \exp \left(-\frac{1}{K u^{2 \alpha}}\right),
\end{aligned}
$$

where the equality in the second line follows from (1.3) and the last inequality follows from Lemma 2.3 (i). To prove the left inequality in (4.2), we use the same argument as in the proof of Theorem 3.2,

$$
\begin{aligned}
P\{T(1) \leq u\} & \geq P\left\{\text { for any } t \text { with }|t|>\frac{u}{2},|X(t)| \geq 1\right\} \\
& =1-P\left\{\exists t \text { with }|t|>\frac{u}{2} \text { such that }|X(t)|<1\right\} \\
& \geq 1-\int_{\mathbf{R}^{d}} \min \left\{1, K\left(\frac{1}{|y|}\right)^{d-\frac{1}{\alpha}}\right\} \cdot p\left(y, \frac{u}{2}\right) d y \\
& \geq \int_{\left\{|y| \geq c_{20}\right\}}\left(\frac{1}{2 \pi}\right)^{\frac{d}{2}} \cdot\left(\frac{2}{u}\right)^{\alpha d} \exp \left(-\frac{|y|^{2}}{2^{1-2 \alpha} u^{2 \alpha}}\right) \cdot\left(1-\frac{K}{|y|^{d-\frac{1}{\alpha}}}\right) d y \\
& \geq c_{21} \int_{\left\{|z| \geq c_{22} / u^{\alpha}\right\}} \exp \left(-\frac{|z|^{2}}{2}\right) d z \\
& \geq \exp \left(-\frac{K}{u^{2 \alpha}}\right) .
\end{aligned}
$$

This proves (4.2).

Since it is not known whether $T(1)$ has a bounded density, we will use the following lemma, which gives some information about the local density of a probability measure.

Lemma 4.2. Given $\lambda_{0}>0$, there exists a positive constant $K=K\left(\lambda_{0}\right)$ with the following property: for any Borel probability measure $\mu$ on $\mathbf{R}$, there exists $x \in$ $\left[\lambda_{0}, 2 \lambda_{0}\right]$ such that

$$
\mu((x-\delta, x+\delta)) \leq K \delta^{\frac{1}{2}} \quad \text { for every } 0<\delta<\frac{1}{8} .
$$


Proof. For $n=1,2, \cdots$, let $\delta_{n}=\frac{1}{n^{3}}$ and let

$$
A_{n}=\left\{x \in\left[\lambda_{0}, 2 \lambda_{0}\right]: \mu\left(\left(x-\delta_{n}, x+\delta_{n}\right)\right) \geq K \delta_{n}^{\frac{1}{2}}\right\},
$$

where $K=K\left(\lambda_{0}\right)>0$ will be chosen later. Let

$$
A=\left\{x \in\left[\lambda_{0}, 2 \lambda_{0}\right]: \mu\left(\left(x-\delta_{n}, x+\delta_{n}\right)\right) \geq K \delta_{n}^{\frac{1}{2}} \text { for some } n=1,2, \cdots\right\} .
$$

Denote the Lebesgue measure on $\mathbf{R}$ by $L_{1}$. Then by Fubini's theorem, we have

$$
\begin{aligned}
L_{1}\left(A_{n}\right) & =L_{1}\left\{x \in\left[\lambda_{0}, 2 \lambda_{0}\right]: \mu\left(\left(x-\delta_{n}, x+\delta_{n}\right)\right) \geq K \delta_{n}^{\frac{1}{2}}\right\} \\
& \leq \frac{\int_{\lambda_{0}}^{2 \lambda_{0}} \mu\left(\left(x-\delta_{n}, x+\delta_{n}\right)\right) d x}{K \delta_{n}^{\frac{1}{2}}} \\
& =\frac{\int_{\lambda_{0}}^{2 \lambda_{0}} d x \int 1_{\left(x-\delta_{n}, x+\delta_{n}\right)}(t) d \mu(t)}{K \delta_{n}^{\frac{1}{2}}} \\
& =\frac{\int d \mu(t) \int_{\lambda_{0}}^{2 \lambda_{0}} 1_{\left(t-\delta_{n}, t+\delta_{n}\right)}(x) d x}{K \delta_{n}^{\frac{1}{2}}} \\
& \leq \frac{2}{K} \delta_{n}^{\frac{1}{2}}=\frac{2}{K} n^{-\frac{3}{2}} .
\end{aligned}
$$

Hence

$$
L_{1}(A) \leq \sum_{n=1}^{\infty} \frac{2}{K} n^{-\frac{3}{2}} .
$$

Take $K=K\left(\lambda_{0}\right)>0$ such that $L_{1}(A) \leq \frac{1}{2} \lambda_{0}$. Then there exist $x \in\left[\lambda_{0}, 2 \lambda_{0}\right]$ such that

$$
\mu\left(\left(x-n^{-3}, x+n^{-3}\right)\right) \leq K n^{-\frac{3}{2}} \quad \text { for every } n=1,2, \cdots .
$$

For any $0<\delta<\frac{1}{8}$, there exists an integer $n$ such that

$$
\frac{1}{(n+1)^{3}} \leq \delta<\frac{1}{n^{3}} \text {. }
$$

By (4.4) we have

$$
\begin{aligned}
\mu((x-\delta, x+\delta)) & \leq \mu\left(\left(x-n^{-3}, x+n^{-3}\right)\right) \\
& \leq K n^{-\frac{3}{2}} \\
& \leq K \delta^{\frac{1}{2}}
\end{aligned}
$$

This proves (4.3).

Now we prove the main result of this section.

Theorem 4.1. Let $X(t)(t \in \mathbf{R})$ be a fractional Brownian motion of index $\alpha$ in $\mathbf{R}^{d}$ and $1<\alpha$ d. Then with probability 1 ,

$$
\liminf _{r \rightarrow 0} \frac{T(r)}{\phi(r)}=\gamma
$$

where $\phi(s)=s^{\frac{1}{\alpha}} /\left(\log \log \frac{1}{s}\right)^{\frac{1}{2 \alpha}}$ and $0<\gamma<\infty$ is a constant depending on $\alpha$ and $d$ only. 
Proof. We start with the easy part and prove that there exists a constant $\gamma_{1}>0$ such that

$$
\liminf _{r \rightarrow 0} \frac{T(r)}{\phi(r)} \geq \gamma_{1} \text { a.s. }
$$

For $k=1,2, \cdots$, let $a_{k}=\exp \left(-\frac{k}{\log k}\right)$ and

$$
A_{k}=\left\{\omega: T\left(a_{k}\right) \leq \lambda \phi\left(a_{k}\right)\right\},
$$

where $\lambda>0$ is a constant to be determined later. Then by the scaling property (4.1) of $T(r)$ and Lemma 4.1, we have, for $k$ large enough,

$$
\begin{aligned}
P\left(A_{k}\right) & =P\left\{T(1) \leq \frac{\lambda}{\left(\log \log \frac{1}{a_{k}}\right)^{\frac{1}{2 \alpha}}}\right\} \\
& \leq \exp \left(-\frac{1}{K \lambda^{2 \alpha}} \log \log \frac{1}{a_{k}}\right) \\
& \leq k^{-\frac{1}{K \lambda^{2 \alpha}}} .
\end{aligned}
$$

Take $\lambda=\gamma_{1}>0$ such that $K \gamma_{1}^{2 \alpha}<1$. Then

$$
\sum_{k=1}^{\infty} P\left(A_{k}\right)<\infty .
$$

By Lemma 2.4 (i), with probability 1 , there exists $k_{0}=k_{0}(\omega)$ such that $k \geq k_{0}$ implies

$$
T\left(a_{k}\right) \geq \gamma_{1} \phi\left(a_{k}\right)
$$

Thus

$$
\liminf _{k \rightarrow \infty} \frac{T\left(a_{k}\right)}{\phi\left(a_{k}\right)} \geq \gamma_{1} \text { a.s. }
$$

Since $T(r)$ and $\phi(r)$ are monotone increasing for small $r$, we have that, for $r>0$ small enough and $a_{k+1} \leq r<a_{k}$,

$$
\frac{T(r)}{\phi(r)} \geq \frac{T\left(a_{k+1}\right)}{\phi\left(a_{k+1}\right)} \cdot \frac{\phi\left(a_{k+1}\right)}{\phi\left(a_{k}\right)} .
$$

Observing that

$$
\lim _{k \rightarrow \infty} \frac{\phi\left(a_{k+1}\right)}{\phi\left(a_{k}\right)}=1
$$

we conclude that

$$
\liminf _{r \rightarrow 0} \frac{T(r)}{\phi(r)} \geq \gamma_{1} \text { a.s. }
$$

This proves (4.6).

In order to prove that there exists a positive constant $\gamma_{2}$ such that

$$
\liminf _{r \rightarrow 0} \frac{T(r)}{\phi(r)} \leq \gamma_{2},
$$

we let $b_{k}=\exp \left(-k^{2}\right), \tau_{k}=k^{\theta} b_{k}^{\frac{1}{\alpha}}$, where $\theta=\frac{3}{\alpha d-1}$, and let $\lambda>0$ be a constant to be determined later. Denote

$$
T\left(b_{k}, \tau_{k}\right)=\int_{|t| \leq \tau_{k}} 1_{B\left(0, b_{k}\right)}(X(t)) d t
$$


Then by Theorem 3.2, we have

$$
\begin{aligned}
P\left\{T\left(b_{k}, \tau_{k}\right) \neq T\left(b_{k}\right)\right\} & \leq P\left\{\exists t \text { such that }|t|>\tau_{k} \text { and }|X(t)| \leq b_{k}\right\} \\
& \leq K\left(\frac{b_{k}}{\tau_{k}^{\alpha}}\right)^{d-\frac{1}{\alpha}} \\
& =K k^{-3} .
\end{aligned}
$$

Hence by Lemma 2.4 (i), with probability 1 , there exists $k_{1}=k_{1}(\omega)$ such that $k \geq k_{1}$ implies

$$
T\left(b_{k}, \tau_{k}\right)=T\left(b_{k}\right)
$$

Let

$$
E_{k}=\left\{T\left(b_{k}, \tau_{k}\right)<\lambda_{k} \phi\left(b_{k}\right)\right\}
$$

where $\lambda \leq \lambda_{k} \leq 2 \lambda$ will be chosen later. By (4.1) and Lemma 4.1, we have

$$
\begin{aligned}
P\left(E_{k}\right) & \geq P\left\{T\left(b_{k}\right)<\lambda_{k} \phi\left(b_{k}\right)\right\} \\
& =P\left\{T(1)<\frac{\lambda_{k}}{\left(\log \log \frac{1}{b_{k}}\right)^{\frac{1}{2 \alpha}}}\right\} \\
& \geq c_{23} k^{-\frac{K}{\lambda^{2 \alpha}}} .
\end{aligned}
$$

Thus, if we take $\lambda=\lambda_{0}$ such that $K / \lambda_{0}^{2 \alpha} \leq 1$, then

$$
\sum_{k=1}^{\infty} P\left(E_{k}\right)=\infty \text {. }
$$

In order to prove

$$
P\left(\limsup _{k \rightarrow \infty} E_{k}\right)=1
$$

it suffices to show that for any $\epsilon>0,(2.5)$ is satisfied.

Fix a positive integer $k$. For $j>k$, we need to estimate

$$
P\left(E_{k} \cap E_{j}\right)=P\left\{T\left(b_{k}, \tau_{k}\right)<\lambda_{k} \phi\left(b_{k}\right), T\left(b_{j}, \tau_{j}\right)<\lambda_{j} \phi\left(b_{j}\right)\right\} .
$$

We denote this probability by $Q$. In order to create independence, we follow [Ta2] and make use of the stochastic integral representation (1.1). We set $v=\sqrt{\tau_{k} \tau_{j}}$ and consider the following two processes:

$$
X^{1}(t)=\left(Y_{1}^{1}(t), \cdots, Y_{d}^{1}(t)\right)
$$

and

$$
X^{2}(t)=\left(Y_{1}^{2}(t), \cdots, Y_{d}^{2}(t)\right),
$$

where $Y_{1}^{1}, \cdots, Y_{d}^{1}$ are independent copies of

$$
Z^{1}(t)=\int_{|x| \leq v} G(t, x) d W(x)
$$


and $Y_{1}^{2}, \cdots, Y_{d}^{2}$ are independent copies of

$$
Z^{2}(t)=\int_{|x| \geq v} G(t, x) d W(x) .
$$

Then $X^{1}$ and $X^{2}$ are independent and $X(t)=X^{1}(t)+X^{2}(t)$. By Lemma 5.2 of $[\mathrm{Ta} 2]$, for any $|t| \leq \tau_{k}$,

$$
\begin{aligned}
\left\|Z^{1}(t)\right\|_{2} & \leq K \sqrt[4]{\tau_{k} \tau_{j}} \cdot \tau_{k}^{\alpha-\frac{1}{2}} \\
& =K \sqrt[4]{\frac{\tau_{j}}{\tau_{k}}} \cdot \tau_{k}^{\alpha},
\end{aligned}
$$

and for any $|t| \leq \tau_{j}$,

$$
\left\|Z^{2}(t)\right\|_{2} \leq K \sqrt{\frac{\tau_{j}}{\tau_{k}}}\left(\tau_{k} \tau_{j}\right)^{\frac{\alpha}{2}}
$$

where $\|Z\|_{2}=\left(E\left(Z^{2}\right)\right)^{\frac{1}{2}}$.

For any $0<\delta<1$, let $\eta>0$ satisfy

$$
\frac{1}{(1-2 \eta)^{\frac{1}{\alpha}}}=1+\delta
$$

These numbers will be fixed for the moment. Clearly $\eta \geq \frac{\alpha}{4} \delta$. Since $\left|X^{1}(t)\right| \leq \eta b_{k}$ and $\left|X^{2}(t)\right| \leq(1-\eta) b_{k}$ imply $|X(t)| \leq b_{k}$, we have

$$
\begin{gathered}
\left\{T\left(b_{k}, \tau_{k}\right)<\lambda_{k} \phi\left(b_{k}\right)\right\} \subseteq\left\{\int_{|t| \leq \tau_{k}} 1_{B\left(0,(1-\eta) b_{k}\right)}\left(X^{2}(t)\right) d t<\lambda_{k} \phi\left(b_{k}\right)\right\} \\
\cup\left\{\max _{|t| \leq \tau_{k}}\left|X^{1}(t)\right|>\eta b_{k}\right\} .
\end{gathered}
$$

Similarly,

$$
\begin{gathered}
\left\{T\left(b_{j}, \tau_{j}\right)<\lambda_{j} \phi\left(b_{j}\right)\right\} \subseteq\left\{\int_{|t| \leq \tau_{j}} 1_{B\left(0,(1-\eta) b_{j}\right)}\left(X^{1}(t)\right) d t<\lambda_{j} \phi\left(b_{j}\right)\right\} \\
\cup\left\{\max _{|t| \leq \tau_{j}}\left|X^{2}(t)\right|>\eta b_{j}\right\} .
\end{gathered}
$$

By (4.13) and (4.14), we have that $Q$ is less than

$$
\begin{gathered}
P\left\{\int_{|t| \leq \tau_{k}} 1_{B\left(0,(1-\eta) b_{k}\right)}\left(X^{2}(t)\right) d t<\lambda_{k} \phi\left(b_{k}\right)\right. \\
\left.\int_{|t| \leq \tau_{j}} 1_{B\left(0,(1-\eta) b_{j}\right)}\left(X^{1}(t)\right) d t<\lambda_{j} \phi\left(b_{j}\right)\right\} \\
+P\left\{\max _{|t| \leq \tau_{k}}\left|X^{1}(t)\right|>\eta b_{k}\right\}+P\left\{\max _{|t| \leq \tau_{j}}\left|X^{2}(t)\right|>\eta b_{j}\right\}
\end{gathered}
$$


By the independence of $X^{1}$ and $X^{2}$, we have

$$
\begin{aligned}
P\left\{\int_{|t| \leq \tau_{k}} 1_{B\left(0,(1-\eta) b_{k}\right)}\left(X^{2}(t)\right) d t<\lambda_{k} \phi\left(b_{k}\right),\right. & \left.\int_{|t| \leq \tau_{j}} 1_{B\left(0,(1-\eta) b_{j}\right)}\left(X^{1}(t)\right) d t<\lambda_{j} \phi\left(b_{j}\right)\right\} \\
= & P\left\{\int_{|t| \leq \tau_{k}} 1_{B\left(0,(1-\eta) b_{k}\right)}\left(X^{2}(t)\right) d t<\lambda_{k} \phi\left(b_{k}\right)\right\} \\
& \cdot P\left\{\int_{|t| \leq \tau_{j}} 1_{B\left(0,(1-\eta) b_{j}\right)}\left(X^{1}(t)\right) d t<\lambda_{j} \phi\left(b_{j}\right)\right\} \\
\leq & P\left\{\int_{|t| \leq \tau_{k}} 1_{B\left(0,(1-2 \eta) b_{k}\right)}(X(t)) d t<\lambda_{k} \phi\left(b_{k}\right)\right\} \\
& \cdot P\left\{\int_{|t| \leq \tau_{j}} 1_{B\left(0,(1-2 \eta) b_{j}\right)}(X(t)) d t<\lambda_{j} \phi\left(b_{j}\right)\right\} \\
& +P\left\{\max _{|t| \leq \tau_{k}}\left|X^{1}(t)\right|>\eta b_{k}\right\}+P\left\{\max _{|t| \leq \tau_{j}}\left|X^{2}(t)\right|>\eta b_{j}\right\} \\
\hat{=} & Q_{1}+Q_{2}+Q_{3} .
\end{aligned}
$$

Hence,

$$
Q \leq Q_{1}+2 Q_{2}+2 Q_{3} .
$$

Now we estimate $Q_{1}, Q_{2}$ and $Q_{3}$ respectively. First we consider

$$
\begin{aligned}
& P\left\{\int_{|t| \leq \tau_{k}} 1_{B\left(0,(1-2 \eta) b_{k}\right.}(X(t)) d t<\lambda_{k} \phi\left(b_{k}\right)\right\} \\
& \leq P\left\{T\left((1-2 \eta) b_{k}\right)<\lambda_{k} \phi\left(b_{k}\right)\right\}+P\left\{T\left((1-2 \eta) b_{k}\right) \neq T\left((1-2 \eta) b_{k}, \tau_{k}\right)\right\} \\
& \leq P\left\{T(1)<\frac{1}{(1-2 \eta)^{\frac{1}{\alpha}}} \cdot \frac{\lambda_{k} \phi\left(b_{k}\right)}{b_{k}^{\frac{1}{\alpha}}}\right\}+K k^{-3},
\end{aligned}
$$

where the last inequality follows from (4.1) and (4.8). By applying Lemma 4.2 to the distribution of $T(1)\left(\log \log \frac{1}{b_{k}}\right)^{1 / 2 \alpha}$, we can choose $\lambda_{k} \in\left[\lambda_{0}, 2 \lambda_{0}\right]$ such that

$$
P\left\{\lambda_{k} \leq T(1)\left(\log \log \frac{1}{b_{k}}\right)^{\frac{1}{2 \alpha}}<(1+\delta) \lambda_{k}\right\} \leq K \delta^{\frac{1}{2}} \quad \text { for any } 0<\delta<\frac{1}{8} .
$$

Hence, by Lemma 4.1 and (4.17), the probability in (4.16) is less than

$$
\begin{aligned}
& P\left\{T(1)<\frac{\lambda_{k} \phi\left(b_{k}\right)}{b_{k}^{\frac{1}{\alpha}}}\right\} \cdot \exp \left(\frac{P\left\{\frac{\lambda_{k} \phi\left(b_{k}\right)}{b_{k}^{\frac{1}{\alpha}}} \leq T(1)<\frac{(1+\delta) \lambda_{k} \phi\left(b_{k}\right)}{b_{k}^{\frac{1}{\alpha}}}\right\}}{P\left\{T(1)<\frac{\lambda_{k} \phi\left(b_{k}\right)}{b_{k}^{\frac{1}{\alpha}}}\right\}}\right) \\
& \leq P\left\{T(1)<\frac{\lambda_{k} \phi\left(b_{k}\right)}{b_{k}^{\frac{1}{\alpha}}}\right\} \cdot \exp \left(K k \delta^{\frac{1}{2}}\right) \\
& \leq\left(P\left\{T\left(b_{k}, \tau_{k}\right)<\lambda_{k} \phi\left(b_{k}\right)\right\}+K k^{-3}\right) \cdot \exp \left(K k \delta^{\frac{1}{2}}\right) .
\end{aligned}
$$


Combining (4.16) and (4.18), we have

$$
\begin{aligned}
& P\left\{\int_{|t| \leq \tau_{k}} 1_{B\left(0(1-2 \eta) b_{k}\right)}(X(t)) d t<\lambda_{k} \phi\left(b_{k}\right)\right\} \\
& \leq\left(P\left\{T\left(b_{k}, \tau_{k}\right)<\lambda_{k} \phi\left(b_{k}\right)\right\}+K k^{-3}\right) \cdot \exp \left(K k \delta^{\frac{1}{2}}\right)+K k^{-3} \\
& \leq P\left\{T\left(b_{k}, \tau_{k}\right)<\lambda_{k} \phi\left(b_{k}\right)\right\}\left(1+\frac{K}{k^{2}}\right) \cdot \exp \left(K k \delta^{\frac{1}{2}}\right) .
\end{aligned}
$$

Similarly, we have

$$
\begin{aligned}
& P\left\{\int_{|t| \leq \tau_{j}} 1_{B\left(0(1-2 \eta) b_{j}\right)}(X(t)) d t<\lambda_{j} \phi\left(b_{j}\right)\right\} \\
& \leq P\left\{T\left(b_{j}, \tau_{j}\right)<\lambda_{j} \phi\left(b_{j}\right)\right\}\left(1+\frac{K}{j^{2}}\right) \cdot \exp \left(K j \delta^{\frac{1}{2}}\right) .
\end{aligned}
$$

It follows from (4.19) and (4.20) that

$$
\begin{gathered}
Q_{1} \leq P\left\{T\left(b_{k}, \tau_{k}\right)<\lambda_{k} \phi\left(b_{k}\right)\right\} P\left\{T\left(b_{j}, \tau_{j}\right)<\lambda_{j} \phi\left(b_{j}\right)\right\} \\
\cdot\left(1+\frac{K}{k^{2}}\right)\left(1+\frac{K}{j^{2}}\right) \exp \left(K j \delta^{\frac{1}{2}}\right) .
\end{gathered}
$$

The same argument as in [Ta2] using (4.11) and (4.12) yields

$$
P\left\{\max _{|t| \leq \tau_{k}}\left|X^{1}(t)\right|>\eta b_{k}\right\} \leq \exp \left(-K \eta^{2} \sqrt{\frac{\tau_{k}}{\tau_{j}}} \frac{1}{k^{2 \theta \alpha} \log \frac{\tau_{k}}{\tau_{j}}}\right)
$$

and

$$
P\left\{\max _{|t| \leq \tau_{j}}\left|X^{2}(t)\right|>\eta b_{j}\right\} \leq \exp \left(-K \eta^{2}\left(\frac{\tau_{k}}{\tau_{j}}\right)^{1-\alpha} \frac{1}{j^{2 \theta \alpha} \log \frac{\tau_{k}}{\tau_{j}}}\right) .
$$

Now we take

$$
0<\beta<\min \left\{\frac{1}{5}, \frac{1}{3}(1-\alpha)\right\}
$$

and

$$
\delta=\left(\frac{\tau_{j}}{\tau_{k}}\right)^{\beta} .
$$

Then, by (4.15), (4.21), (4.22) and (4.23), we have

$$
\begin{aligned}
Q \leq & P\left(E_{k}\right) P\left(E_{j}\right)\left(1+\frac{K}{k^{2}}\right)\left(1+\frac{K}{j^{2}}\right) \exp \left(K j\left(\frac{\tau_{j}}{\tau_{k}}\right)^{\frac{\beta}{2}}\right)+c_{24} P\left(E_{k}\right) \\
& \cdot\left\{k \exp \left(-\frac{K}{k^{2 \theta \alpha} \log \frac{\tau_{k}}{\tau_{j}}}\left(\frac{\tau_{k}}{\tau_{j}}\right)^{\frac{1}{2}-2 \beta}\right)+k \exp \left(-\frac{K}{j^{2 \theta \alpha} \log \frac{\tau_{k}}{\tau_{j}}}\left(\frac{\tau_{k}}{\tau_{j}}\right)^{1-\alpha-2 \beta}\right)\right\} .
\end{aligned}
$$

By the choice of $\tau_{k}$, we have

$$
\frac{\tau_{k}}{\tau_{j}}=\left(\frac{k}{j}\right)^{\theta} \exp \left(\frac{1}{\alpha}\left(j^{2}-k^{2}\right)\right) .
$$


Hence, by (4.24), for any $\epsilon>0$, there exist a constant $K>0$ and a positive integer $k_{0}$ such that for any $k \geq k_{0}$ and any $J>k$, we have

$$
\sum_{j=k+1}^{J} P\left(E_{k} \cap E_{j}\right) \leq P\left(E_{k}\right)\left(K+(1+\epsilon) \sum_{j=k+1}^{J} P\left(E_{j}\right)\right) .
$$

It follows from (4.10), (4.25) and Lemma 2.4 that

$$
P\left(\limsup _{k \rightarrow \infty} E_{k}\right) \geq \frac{1}{1+2 \epsilon} .
$$

Since $\epsilon>0$ is arbitrary, we conclude that

$$
P\left\{T\left(b_{k}\right)<\lambda_{k} \phi\left(b_{k}\right) \text { i. o. }\right\}=1 .
$$

Therefore, with probability 1 ,

$$
\liminf _{r \rightarrow 0} \frac{T(r)}{\phi(r)} \leq \gamma_{2},
$$

where $\gamma_{2}=2 \lambda_{0}$. By (4.5), (4.26) and the zero-one law in [PT], we obtain (4.5). This completes the proof of Theorem 4.1.

Since for any $t_{0} \in \mathbf{R}, X\left(t+t_{0}\right)-X\left(t_{0}\right)(t \in \mathbf{R})$ is also a fractional Brownian motion of index $\alpha$, we have the following corollary.

Corollary 4.1. Let $X(t)(t \in \mathbf{R})$ be a fractional Brownian motion of index $\alpha$ in $\mathbf{R}^{d}$ and $1<\alpha d$. Then for any $t_{0} \in \mathbf{R}$, with probability 1 ,

$$
\liminf _{r \rightarrow 0} \frac{T_{X\left(t_{0}\right)}(r)}{\phi(r)}=\gamma .
$$

\section{Packing Measure of Fractional Brownian Motion}

We are ready to calculate the packing measure of a transient fractional Brownian motion in $\mathbf{R}^{d}$.

Proposition 5.1. Let $X(t)(t \in \mathbf{R})$ be a fractional Brownian motion in $\mathbf{R}^{d}$ of index $\alpha$. If $1<\alpha d$, then there exist positive constants $C_{1}$ and $C_{2}$ such that, with probability 1 ,

$$
C_{1} \leq \phi-p(X([0,1])) \leq C_{2},
$$

where $\phi(s)=s^{\frac{1}{\alpha}} /\left(\log \log \frac{1}{s}\right)^{\frac{1}{2 \alpha}}$.

Proof. We define a random Borel measure $\mu$ on $X([0,1])$ as follows. For any Borel set $B \subseteq \mathbf{R}^{d}$, let

$$
\mu(B)=L_{1}\{t \in[0,1], X(t) \in B\} .
$$

Then $\mu\left(\mathbf{R}^{d}\right)=\mu(X([0,1]))=1$. By Corollary 4.1 , for each fixed $t_{0} \in(0,1)$, with probability 1

$$
\begin{aligned}
\liminf _{r \rightarrow 0} & \frac{\mu\left(B\left(X\left(t_{0}\right), r\right)\right)}{\phi(r)} \\
\leq & \liminf _{r \rightarrow 0} \frac{T_{X\left(t_{0}\right)}(r)}{\phi(r)}=\gamma .
\end{aligned}
$$


Let $E(\omega)=\left\{X\left(t_{0}\right): t_{0} \in(0,1)\right.$ and (5.2) holds $\}$. Then $E(\omega) \subseteq X([0,1])$. A Fubini argument shows $\mu(E(\omega))=1$, a.s. Hence by Lemma 2.1, we have

$$
\phi-p(E(\omega)) \geq K_{1}^{-3} \gamma^{-1} \text {. }
$$

This proves the left hand inequality of (5.1) with $C_{1}=K_{1}^{-3} \gamma^{-1}$.

To prove the right hand inequality of $(5.1)$, we let $\mathcal{J}_{k}$ be the family of dyadic intervals of length $2^{-k}$ in $[0,1]$. For each $x \in[0,1]$, let $I_{k}(x)$ be the dyadic interval in $\mathcal{J}_{k}$ which contains $x$ and let

$$
a_{k}(x)=\sup _{s, t \in I_{k}(x)}|X(t)-X(s)| .
$$

For any $k \geq 1$ and any $I \in \mathcal{J}_{k}$, by Lemma 2.2 , we have

$$
P\left\{\sup _{s, t \in I}|X(t)-X(s)| \geq u 2^{-k \alpha}\right\} \leq \exp \left(-\frac{u^{2}}{K}\right) .
$$

Take $u=\sqrt{K \lambda \log k}$, where $\lambda>6+\frac{1}{2 \alpha}$. We have

$$
P\left\{\sup _{s, t \in I}|X(t)-X(s)| \geq \sqrt{K \lambda \log k} 2^{-k \alpha}\right\} \leq \frac{1}{k^{\lambda}} .
$$

Denote

$$
M_{k}=\#\left\{I \in \mathcal{J}_{k}, \quad \sup _{s, t \in I}|X(t)-X(s)| \geq \sqrt{K \lambda \log k} 2^{-k \alpha}\right\} ;
$$

then

$$
P\left\{M_{k} \geq 2^{k} \cdot \frac{1}{k^{\lambda-5}}\right\} \leq \frac{1}{k^{5}} .
$$

By Lemma 2.4 (i), with probability one, for $k$ large enough,

$$
M_{k}<\frac{2^{k}}{k^{\lambda-5}} .
$$

Let $\Omega_{0}$ be the event that (5.3) holds eventually, and let $\Omega_{1}$ be the event that (2.5) holds. Then $P\left(\Omega_{0} \cap \Omega_{1}\right)=1$. Fix an $\omega \in \Omega_{0} \cap \Omega_{1}$, and let $k_{0}=k_{0}(\omega)$ be a positive integer such that $k \geq k_{0}$ implies (5.3).

For any $0<\epsilon<2^{-k_{0}}$ and any $\epsilon$-packing $\left\{\bar{B}\left(X\left(t_{i}\right), r_{i}\right)\right\}$ of $X([0,1])$, we will show that for some absolute constant $C_{2}>0$,

$$
\sum_{i} \phi\left(2 r_{i}\right) \leq C_{2} \text { a.s. }
$$

For each $i$, let

$$
k_{i}=\inf \left\{k: a_{k}\left(t_{i}\right) \leq r_{i}\right\} .
$$

Thus $a_{k_{i}-1}\left(t_{i}\right)>r_{i}$ and the $\left\{I_{k_{i}}\left(t_{i}\right)\right\}$ are disjoint, so

$$
\sum_{i} 2^{-k_{i}} \leq 1
$$

Let $\Gamma_{1}$ be the set of the positive integer $i$ 's with

$$
a_{k_{i}-1}\left(t_{i}\right) \leq \sqrt{K \lambda \log \left(k_{i}-1\right)} 2^{-\left(k_{i}-1\right) \alpha} .
$$


By (5.5), we obtain

$$
\begin{aligned}
\sum_{i \in \Gamma_{1}} \phi\left(2 a_{k_{i}-1}\left(t_{i}\right)\right) & \leq c_{25} \sum_{i} 2^{-k_{i}} \\
& \leq c_{25} .
\end{aligned}
$$

Let $\Gamma_{2}$ be the set of the $i$ 's satisfying

$$
a_{k_{i}-1}\left(t_{i}\right) \geq \sqrt{K \lambda \log \left(k_{i}-1\right)} 2^{-\left(k_{i}-1\right) \alpha} .
$$

By (5.3) and the uniform modulus of continuity of $X$, we have

$$
\begin{aligned}
\sum_{i \in \Gamma_{2}} \phi\left(2 a_{k_{i}-1}\left(t_{i}\right)\right) & \leq \sum_{i \in \Gamma_{2}} \frac{2^{k_{i}-1}}{\left(k_{i}-1\right)^{\lambda-5}} \cdot \phi\left(K \sqrt{k_{i}-1} 2^{-\alpha\left(k_{i}-1\right)}\right) \\
& \leq c_{26} \sum_{k=1} \frac{1}{k^{\lambda-5-\frac{1}{2 \alpha}}}=c_{27} .
\end{aligned}
$$

Combining (5.6) and (5.7), we have

$$
\sum_{i} \phi\left(2 a_{k_{i}-1}\left(t_{i}\right)\right) \leq c_{25}+c_{27}
$$

This implies (5.4), and by (2.2) we have

$$
\phi-P(X([0,1])) \leq C_{2} .
$$

The right hand inequality of (5.1) follows from (2.4).

Since fractional Brownian motion $X(t)(t \in \mathbf{R})$ is ergodic (cf. [T]), the same proof as that of Proposition 5.1 in $[\mathrm{T}]$ yields the following theorem.

Theorem 5.1. Let $X(t)(t \in \mathbf{R})$ be a fractional Brownian motion of index $\alpha$ in $\mathbf{R}^{d}$. If $1<\alpha d$, then there exists a constant $K, 0<K<\infty$, such that, with probability 1 ,

$$
\phi-p(X([0, t])=K t \quad \text { for any } t>0,
$$

where $\phi(s)=s^{\frac{1}{\alpha}} /\left(\log \log \frac{1}{s}\right)^{\frac{1}{2 \alpha}}$.

\section{ACKNOWLEDGEMENT}

I am very much indebted to Professor Talagrand for his valuable suggestions and discussions. Thanks are also due to the referee for his careful reading of the first version of this paper.

\section{REFERENCES}

[CT] Ciesielski, Z. and Taylor, S. J., First passage times and sojourn times for Brownian motion in space and the exact Hausdorff measure of the sample path. Trans. Amer. Math. Soc. 103 (1962), 434 - 450. MR 26:816

[DE] Dvoretzky, A. and Erdös, P., Some problems on random walk in space. Proc. Second Berkeley Sympos., Univ. of California Press, Berkeley, 1950, 353-367. MR 13:852b

[F] Falconer, K. J., Fractal Geometry - Mathematical Foundations And Applications. Wiley \& Sons, 1990. MR 92j:28008

[FT] Fristedt, B. E. and Taylor, S. J., The packing measure of a general subordinator. Prob. Th. Rel. Fields 92 (1992), 493 - 510. MR 93e:60150

[K] Kahane, J-P., Some Random Series of Functions. 2nd edition. Cambridge University Press, 1985. MR 87m:60119 
[Ka] Kakutani, S., On Brownian motion in n-space. Proc. Imperial Acad. Tokyo 20 (1944), 648 - 652. MR 7:315a

[LT] Ledoux, M. and Talagrand, M., Probability in Banach Spaces. Springer Verlag, 1991. MR 93c:60001

[LeT] LeGall, F. and Taylor, S. J., The packing measure of planar Brownian motion. Progress in Probability and Statistics. Seminar on Stochastic Processes. Birkhäuser, Boston, 1986, 139 - 147. MR 89a:60189

[M] Marcus, M. B., Hölder conditions for Gaussian processes with stationary increments. Trans. Amer. Math. Soc. 134 (1968), 29 - 52. MR 37:5930

[P] Pitt, L. D. Local times for Gaussian random fields. Indiana Univ. Math. J. 27 (1978), 309 - 330. MR 57:10796

[PT] Pitt, L. D. and Tran, L. T., Local sample path properties of Gaussian fields. Ann. of Probab. 7 (1979), 477 - 493. MR 80g:60035

[PS] Port, S. C. and Stone, C. J., Brownian Motion and Classical Potential Theory. Academic Press, New York, 1978. MR 58:11459

[RT] Rezakhanlou, F. and Taylor, S. J., The packing measure of the graph of a stable process. Astérisque 157-158 (1988), 341 - 361. MR 90h:60039

[ST] Saint Raymond, X. and Tricot, C., Packing regularity of sets in $n$-space. Math. Proc. Camb. Phil. Soc. 103 (1988), 133 - 145. MR 88m:28002

[T] Takashima, K., Sample path properties of ergodic self-similar processes. Osaka J. Math. 26 (1989), 159 - 189. MR 90e:60056

[Ta1] Talagrand, M., Hausdorff measure of trajectories of multiparameter fractional Brownian motion. Ann. of Probab. 23 (1995) 767 - 775. CMP 1995:13

[Ta2] Talagrand, M., Lower classes for fractional Brownian motion. J. Theoret Probab. 9 (1996) 191-213.

[Tay1] Taylor, S. J., The use of packing measure in the analysis of random sets. Lecture Notes in Math. 1203 (1986), 214 - 222. MR 88i:60078

[Tay2] Taylor, S. J., The measure theory of random fractals. Math. Proc. Camb. Phil. Soc. 100 (1986), 383 - 406. MR 87k:60189

[TT] Taylor, S. J. and Tricot, C. Packing measure and its evaluation for a Brownian path. Trans. Amer. Math. Soc. 288 (1985), 679 - 699. MR 87a:28002

Department of Mathematics, The Ohio State University, Columbus, Ohio 43210

Current address: Department of Mathematic, University of Utah, Salt Lake City, Utah 84112 